\title{
Scale Invariant Gabor Descriptor-Based Noncooperative Iris Recognition
}

\author{
Yingzi Du, Craig Belcher, and Zhi Zhou \\ Department of Electrical and Computer Engineering, Indiana University-Purdue University Indianapolis, \\ Indianapolis, IN 46202, USA \\ Correspondence should be addressed to Yingzi Du, yidu@iupui.edu
}

Received 1 January 2010; Accepted 18 March 2010

Academic Editor: Robert W. Ives

Copyright ( 2010 Yingzi Du et al. This is an open access article distributed under the Creative Commons Attribution License, which permits unrestricted use, distribution, and reproduction in any medium, provided the original work is properly cited.

A new noncooperative iris recognition method is proposed. In this method, the iris features are extracted using a Gabor descriptor. The feature extraction and comparison are scale, deformation, rotation, and contrast-invariant. It works with off-angle and lowresolution iris images. The Gabor wavelet is incorporated with scale-invariant feature transformation (SIFT) for feature extraction to better extract the iris features. Both the phase and magnitude of the Gabor wavelet outputs were used in a novel way for local feature point description. Two feature region maps were designed to locally and globally register the feature points and each subregion in the map is locally adjusted to the dilation/contraction/deformation. We also developed a video-based noncooperative iris recognition system by integrating video-based non-cooperative segmentation, segmentation evaluation, and score fusion units. The proposed method shows good performance for frontal and off-angle iris matching. Video-based recognition methods can improve non-cooperative iris recognition accuracy.

\section{Introduction}

Performing noncooperative iris recognition is important for a number of tasks, such as video surveillance and watchlist monitoring (identifying most wanted criminals/suspects) [1-4]. In addition, noncooperative iris recognition systems can provide added convenience for cooperative users for identification [5]. However, it is challenging to design an iris recognition system that can work in a noncooperative situation, where the image quality may be low and the eye may be deformed, due to a nonfrontal gaze.

In recent years, several methods have been developed for iris recognition $[2,3]$. Most of these methods are designed for frontal and high-quality iris images. Among them, Daugman's approach has been most widely used in the commercialized iris recognition systems [6-9]. This method transforms the segmented iris image into log-polar coordinates, extracts the iris features using a 2D Gabor wavelet, and encodes the phase information into a binary iris code $[7,9]$. Hamming distance is used to match two iris codes [7]. Daugman's method has been tested and evaluated using large databases, such as the United Arab Emirates (UAE) database with over 600,000 iris images with over 200 billion comparisons [8]. Chen et al. proposed using Daugman's 2D Gabor filter with quality measure enhancement to improve the recognition accuracy [10]. Matey et al. used Daugman's method in their Iris on the Move (IOM) system [11] with better optics and illumination to perform iris recognition at a distance. Masek and Kovesi found that Gabor wavelets can have a DC component and proposed using a 1D LogGabor filter [12]. Ma et al. proposed using a 2D filter similar to the Gabor filter [13]. Other works include Wildes [14], who proposed use of a Laplacian pyramid to decompose the iris features for matching. Sudha et al. [15, 16] proposed using edge maps to extract iris patterns and using Hausdorff distance for pattern matching. Boles and Boashash proposed using normalized wavelet transform zero-crossings [17]. Sun et al. proposed using moment-based iris blob matching [18]. Hollingsworth et al. proposed using the "best bits" in the middle band to improve the recognition accuracy [19]. Thornton et al. proposed using correlation filters with Bayesian deformation estimation [20]. Du et al. proposed using 1D Local Texture Patterns [21]. Velisavljevic used oriented separable wavelet transforms to do iris feature 
extraction [22]. Miyazawa et al. used a 2D Fourier Phase Code (FPC) method for representing iris information [23]. Tajbakhsh et al. used both visible light and near infrared iris images for iris recognition [24]. None of these methods are designed for nonideal situations.

In the past, several researchers have worked on non-ideal iris recognition $[25,26]$. Proenca and Alexandre $[5,27]$ have worked on frontal iris recognition under visible wavelengths using the UBIRIS database [28]. Compared to NIR images, such as CASIA [29] and ICE [30] databases, there is more reflection noise in the visible wavelength iris images. Vatsa et al. applied a set of selected quality local enhancement algorithms to generate a single high-quality iris image for iris recognition [31]. In [32], we added several modules to help the traditional iris recognition system work in nonideal situations. However, all of these methods are designed for frontal-gaze iris images.

For nonfrontal iris recognition, Daugman proposed using Fourier-based trigonometry to estimate the two spherical components of angle of gaze and used an affine transformation to "correct" the image and center the gaze [9]. Schuckers et al. proposed two methods to calculate angle of gaze: using Daugman's integrodifferential operator and also an angular deformation calibration model [33]. It is assumed that an estimate of the degree of off-angle is available for the algorithms and subjects are required to place their heads on a chin rest looking front (while the camera is rotated horizontally in fixed angles). Both methods are limited because "the affine transformation assumes the iris is planar, whereas in fact it has some curvature" [9].

Recently, we proposed the Regional Scale Invariant Feature Transform (SIFT) approach [34] for noncooperative iris recognition which works for off-angle iris images. Iris features are described without a polar or affine transformation and the feature point descriptors are scale and rotation invariant. However, the iris region consists of both noise and patterns, and Regional SIFT describes the area around a feature point using gradient information, which is not best suited for feature extraction. Most importantly, Regional SIFT would not work well with local pattern deformation.

If the strengths of SIFT and Gabor wavelets can be combined for feature extraction, it may improve the recognition accuracy for off-angle iris images. A simple combination of SIFT and Gabor wavelet method would not work, and it is challenging to design a method that can take advantage of the SIFT and Gabor wavelet. (1) The SIFT method may select many feature points in a small region. This increases the computational complexity. More importantly, the heavy overlapping of the feature descriptors could result in extremely high weighting for the matching results in the small region. (2) The eyes are deformed in the off-angle images. The global Gabor wavelet parameters would not work for off-angle iris images. This means that we need to design localized Gabor wavelets. How to design the Gabor wavelets to locally describe the feature points is a challenge. More importantly, how does one design the approach to ensure local deformation invariance? (3) In the SIFT method, the matching of the feature points does not incorporate global information. In iris recognition, the feature location is an important piece of information in performing the matching. How does one incorporate the global information in the matching to ensure accurate recognition results? (4) For off-angle iris images, the image segmentation is a challenge. How does one design a recognition method that can be tolerant of small segmentation error?

It would be desirable if an iris recognition algorithm can have the following capabilities:

(i) perform iris recognition for both frontal and offangle iris images,

(ii) be scale invariant in both local and global levels,

(iii) extract iris features efficiently and locally even under the deformation situation,

(iv) be tolerant of segmentation error.

The goal of our work is to design such an iris recognition algorithm. The proposed research effort has four novelties as compared to the previous works. (1) To better extract the iris features, we incorporated Gabor wavelets with SIFT for feature extraction. (2) Both the phase and magnitude of the Gabor wavelet output are weighted and fused in a novel way for local feature point description. (3) To compensate for the iris local deformation due to dilation/contraction and off-angle image acquisition, provide global registration information of the feature points, and improve the matching efficiency, we used two feature region-based methods and ensured that each subregion was locally adjusted to the dilation/contraction/eye deformation. In this paper, we propose two subregion maps for each image for feature point detection. (4) To be tolerant of the segmentation error, we allowed the feature point from one image to match with feature points in its nearby locations from another image.

The rest of the paper is organized as follows. In Section 2, we give a brief review of the SIFT-based methods and discuss why these methods could not work in noncooperative iris recognition. In Section 3, we introduce the proposed Gabor descriptor method and provide technical details about how to develop the feature subregion maps, select the feature points, describe each feature point, and match feature points from different images. The experimental results, comparisons with the state-of-the-art algorithms, and discussions are reported in Section 4. Section 5 describes the proposed video-based noncooperative iris recognition system and discusses the implementation results. Section 6 draws some conclusions.

\section{Brief Review of SIFT-Based Methods}

Local descriptor-based methods are widely used. Local descriptors computed for interest regions are distinctive, robust to occlusion, and (sometimes) do not require segmentation [35]. Lowe [36] proposed the scale invariant feature transformation (SIFT) method, which describes an object as a group of feature points such that the object can be found in an image with invariance to scale, rotation, and affine transformations. The SIFT features are local and based on the appearance of the object at particular interest points, 


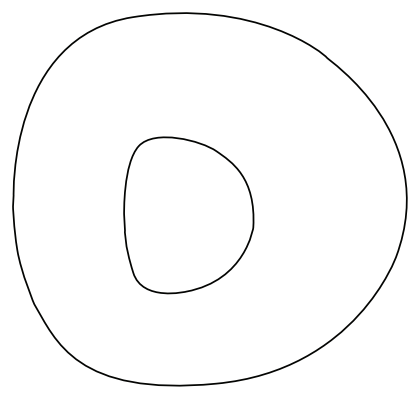

(a) Iris area

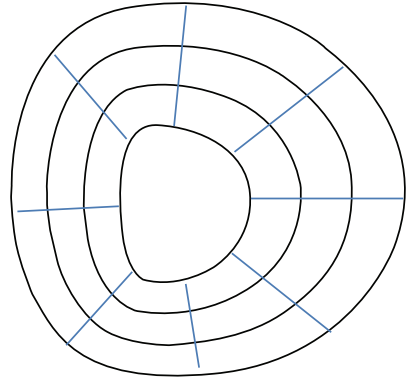

(b) Subregion map 1

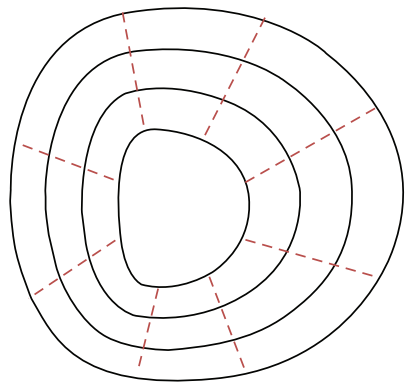

(c) Subregion map 2

Figure 1: Demonstration of the iris subregion selection. (Note that there are more subregions than shown here.) The subregion map 2 is about half angular resolution shifting of subregion map 1.

and are invariant to image scale and rotation. This method has been widely used in current object recognition. In [37], Ke and Sukthankar applied Principal Components Analysis (PCA) to the normalized image gradient patch. Carneiro and Jepson [38] proposed a phase-based descriptor. In [35], Mikolajczyk and Schmid proposed the gradient location and orientation histogram (GLOH), which is an extension of the SIFT descriptor. In [39], Cheng et al. proposed using a multiple support region based SIFT approach for deformable image recognition. In [40], Mortensen et al. proposed SIFT method with global context vector that adds curvilinear shape information from a much larger neighborhood. Some other local descriptor methods include geodesic intensity histogram [41], spin images [42, 43], shape context [44-46], and steerable filter [47-50]. Recently, Lepetit and Fua [51] proposed to use training to find the most repeatable object keypoints. The application of this method is very limited because it requires the training images have a well-registered target object, which is often difficult in real-life scenarios.

In [52], Quelhas et al. used a SIFT-like method to select the feature points and used bag-of-visterms (extended concept of bag-of-visual-words ) to model the local features. Bag-of-visual-words (BoV) model is analogous to the bagof-words (BoW) model in natural language processing and information retrieval [53-56], where a text is represented as an unordered collection of words, while grammar and even word order was disregarded. The BoV in object recognition used a similar concept to describe the local patterns using feature vectors. A codebook of the features is generated by offline quantization of local descriptors. The challenge in $\mathrm{BoV}$ is to find general and representative feature vectors that can describe the local pattern and training is often necessary [57-59]. The BoV did not take the spatial relationships into consideration, which introduces ambiguity in object recognition. This hybrid model by Quelhas et al. [52] can largely improve the recognition accuracy over using $\mathrm{BoV}$ model alone. However, similar to general $\mathrm{BoV}$, it is a challenge to perform robust object recognition.

All the above methods are designed for general object feature extraction, and did not take the iris features into account. In [34], we proposed region-based SIFT approach to improve the recognition accuracy over the traditional SIFT method. However, as we discussed in Section 1, this method

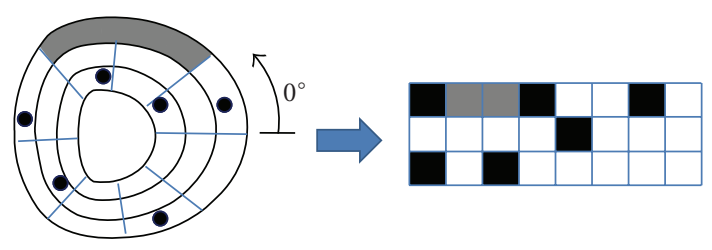

(a) Masked feature point map for feature subregion map 1

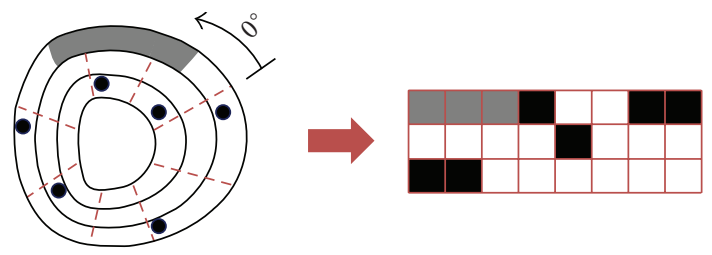

(b) Masked feature point map for feature subregion map 2

Figure 2: Masked Feature Point Map. (Note that these images are for illustration only and do not show the full 720 bins used.) White regions do not contain any feature points. Black regions have one feature point each. The gray regions are noisy regions that should not be used in matching.

has its own limitations. In [9], Daugman has used 2D Gabor wavelet for iris recognition and showed that it worked very well in iris pattern extraction. If the strengths of both local descriptor and Gabor wavelet can be combined in feature extraction, we can design a better local descriptor.

\section{Gabor Descriptor-Based Off-Angle Iris Recognition}

To perform iris recognition, the first step is to segment the iris images. The segmentation of an off-angle iris image itself is a challenging problem $[11,27,31,60]$. In [61], we designed a video-based noncooperative iris segmentation method. In this research, we use the general conic model in [61] for offangle image segmentation. In this paper, our focus is how to design the iris feature extraction and matching method. Our proposed method includes the following steps: feature point selection, feature description, and region-based matching. 

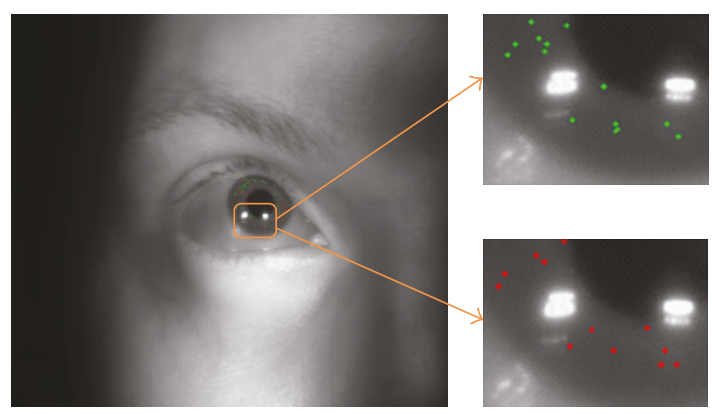

Figure 3: Stable feature points found in two subregion maps in a real image. The left image is an original image with feature points. The top right and bottom right images show the feature points detected in subregion map 1 and subregion map 2, respectively.

3.1. Feature Subregion Maps. The SIFT method finds many points stable within scale space with many points possible in a very small region. The goal of our approach is to increase the opportunity to correctly match feature points within a similar relative position with respect to the pupil across multiple iris images; therefore, we should have a small number of features in small areas. More importantly, iris patterns have their own special characteristics, which need to be considered when designing the feature extraction method, (1) The spatial correlations in iris patterns are important in recognition. Therefore, in designing the effective local descriptor method, we should have global information of feature points. (2) The pupil may dilate or contract. For offangle eyes, the distance between the pupil boundary and the limbic boundary would not be uniform. As a result, the iris patterns can be deformed locally. It is important to have some local normalization process in selecting feature points. (3) There could be noisy regions in an iris area, such as eyelids, eyelashes, and glare. These areas should be identified and removed in matching.

In this research, we divide the iris area into a fixed number of subregions, and ensure each region has at most one feature point. In this way, for each feature point, we know its subregion and the correlation of the subregion to other subregions. Therefore, we have the global information of the feature points for matching.

To take local deformation into account, we should not make the subregions to be same size; rather, we should take eye deformation information into account when assigning the subregions. We know that deformation of the eye would change the iris ring radius (the distance between the pupil and limbic boundaries in radius direction). Especially for off-angle eyes, the radius distance is nonuniform over the angular direction. In this research, we assign $x$ bins in radius direction and $y$ bins from 0 to $2 \pi$ in the angular direction, where $x$ represents the resolution in radius direction, and $y$ represents the resolution in the angular direction of the region. It is important to select proper $x$ and $y$ sizes to ensure the efficiency in matching and accuracy in feature extraction.

Total, we will have $x^{*} y$ subregions in the entire iris region. The research results by Daugman in [7] have shown that 8 rings can work well with iris recognition. To include

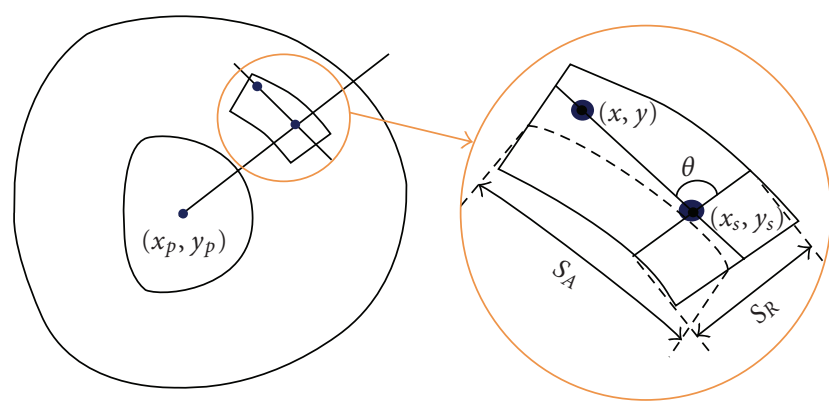

FIGURE 4: The description of $S_{R}, S_{A}, \theta,(x, y)$, and $\left(x_{p}, y_{p}\right)$.

the tolerance of segmentation error of the pupil boundary region and the limbic region (i.e., adding 2 more regions in the radius direction), we use $x=10$ in our research. In the angular direction, by our observation, we found that resolution of the iris patterns are usually within 5 degrees. Therefore, we select $y=72$. In this way, a normalized map of size 10 by 72 is formed. For each sub region, it can only have one feature point. In Section 3.2, we will discuss how to ensure at most one feature point per subregion.

There could be noise (such as eyelids, eyelashes, and glares) in an iris area. It would be important to mask these noisy regions. Therefore, we have three kinds of subregions: noisy regions, regions with one feature point each, and regions without feature points.

The head may tilt and the start point of the subregion map became arbitrary in terms of particular iris patterns. As a result, some iris patterns may cross two subregions in the angular direction. To take this into consideration, we created an additional feature subregion map with the offset of half of the angular resolution, that is, 2.5 degree offset (Figure 1). In this way, if the feature point happens to be in the edge of a subregion in one feature subregion map due to tilt, it will be in the middle of the subregion of the feature subregion map.

3.2. Feature Point Selection. In this research, we used the SIFT Difference of Gaussian (DoG) approach to find potential feature points [36]. However, as we discussed inSection 3.1, it is important to combine the selection of candidate points with the feature subregion maps.

Below is a brief description of the candidate feature points selection process using SIFT feature point selection approach [36]. To find stable feature points, the first step is to apply a nominal Gaussian blur, (1), resulting in $I(x, y)$,

$$
G(x, y)=\frac{1}{2 \pi \sigma_{n}^{2}} e^{-\left(x^{2}+y^{2}\right) / 2 \sigma_{n}{ }^{2}} .
$$

Here $\sigma_{n}=0.5$. Then, the nominally blurred image, $I(x, y)$, is progressively Gaussian blurred. The first Gaussian image is created using

$$
g_{\sigma}=\sqrt{\sigma_{0}^{2}+\sigma_{n}^{2}}
$$

where $\sigma_{0}=1.5 \sqrt{2}$ so that

$$
G(x, y, 1)=G_{g_{\sigma}} * I(x, y) .
$$




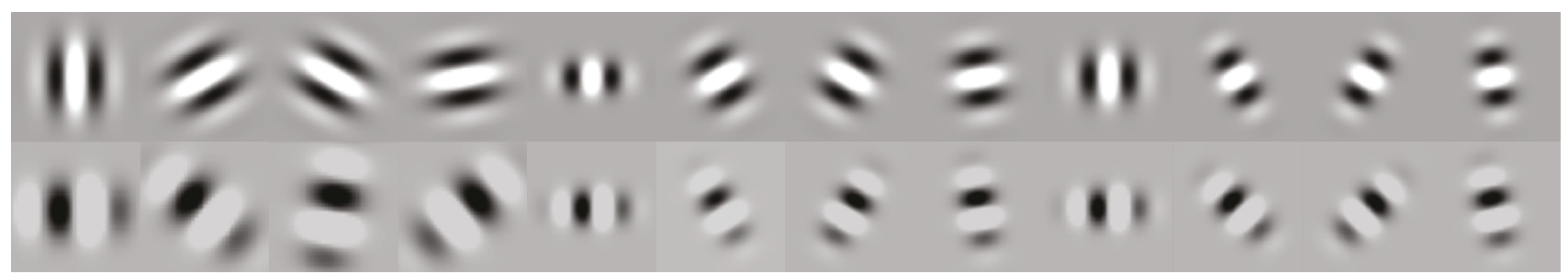

Figure 5: Examples of odd and even Gabor filters with different sizes and orientations. The first row is even filters and the second row is odd filters.

The remaining Gaussian images are created using

$$
\sigma=1.5(\sqrt{2})^{m} \quad(m=0,1,2,3)
$$

resulting in five Gaussian blurred images $(G(x, y, s)(s=$ $0, \ldots, 4)$ ). The size of the Gaussian filter is always the closest odd number to $3 \sigma$. These parameters were selected empirically and are the same for all images. Then the four DoG images are created by subtracting each Gaussian image from the previous Gaussian image in scale:

$$
D(x, y, s)=G(x, y, s+1)-G(x, y, s) \quad(s=0,1,2,3) .
$$

In this research we only use the layers $s=1$ and 2 .

Unlike the general SIFT approach, we only allow one feature point to be selected per layer. For $D(x, y, 1)$ and $D(x, y, 2)$, the local minima and maxima with the highest magnitude of all the points contained within the subregion is stored so that every subregion contains two potential feature points, one scale apart, unless some portion of the subregion is occluded or masked due to noise.

For illustration purposes, Figure 2 shows an example of how the iris area can be divided into multiple subregions with subregions that include occluded pixels (eyelids, eyelashes, or glare) being masked entirely. Since the pupil and limbic boundaries are modeled as ellipses, the sizes of these subregions vary in the radial direction for each of the 72 angular bins. This is a major difference from the previous Regional SIFT method in that the entire iris area can potentially have feature points and every bin size changes with dilation. For further illustration, Figure 2 shows how some bins will contain a feature point corresponding to a point in the annular iris region, whereas others will not. (Note that for ease of viewing, Figure 2 does not show the full 720 bins used.) In addition, to compensate for feature points that are on the boundaries of subregions, a second 10 by 72 normalized feature point map is created with a 2.5 degree angular offset. Note: the two subregions may have different feature points.

Once potential feature points are identified and mapped to the feature point map, the $3 \mathrm{D}$ quadratic method is used to eliminate unstable feature points. Using the Taylor expansion (up to the quadratic terms) of the DoG images, $D(x, y, s)$, shifted so that the origin is at the selected point.

$$
D(\Delta \bar{x})=D+\frac{\partial D^{T}}{\partial \bar{x}} \Delta \bar{x}+\frac{1}{2}(\Delta \bar{x})^{T} \frac{\partial^{2} D}{\partial \bar{x}^{2}} \Delta \bar{x},
$$

where $D$ and its derivatives are evaluated at the selected point and $\Delta \bar{x}=(\Delta x, \Delta y, \Delta s)^{T}$ is the offset from this point. Taking the derivative of this function with respect to $\bar{x}$ and setting it equal to zero, we determine the extremum, $\Delta \tilde{x}$, to be

$$
\Delta \tilde{x}=-\frac{\partial^{2} D^{-1}}{\partial \bar{x}^{2}} \frac{\partial D}{\partial \bar{x}} .
$$

To reject points that have low contrast

$$
D(\Delta \tilde{x})=D+\frac{1}{2} \frac{\partial D^{T}}{\partial \tilde{x}} \tilde{x} .
$$

If $|D(\Delta \tilde{x})|$ is less than 0.03 for a given extrema point, that point is rejected.

To determine if an extrema point is along an edge, the Hessian matrix is used [18],

$$
H=\left[\begin{array}{ll}
D_{x x} & D_{x y} \\
D_{x y} & D_{y y}
\end{array}\right]
$$

where $D$ is the second partial derivative of the DoG image $D(x, y, s)$ at a scale $s$, the following inequality is used to find edge and corner points. If

$$
\frac{\operatorname{Tr}(H)^{2}}{\operatorname{Det}(H)}<\frac{(r+1)^{2}}{r},
$$

the extrema point is considered to be a corner; otherwise, the point is rejected as an edge point. Here $r=10$.

After rejecting points based on contrast, edge value, and stability, the remaining points are assigned a description. However, if in one subregion, there are still 2 feature points available (one feature point per scale), we will then choose the more dominate one (i.e., the one with higher $|D(\Delta \widetilde{x})|$ value). In this way, we ensure that each subregion can have at most one feature point. As we discussed in Section 3.1, we will have 2 feature subregion maps per iris. This means that we will have 2 sets of feature points per iris. Figure 3 shows an example of stable feature points found for an iris in two subregion maps.

3.3. Feature Description. For each feature point, a feature description of length 64 is created based on the normalized and Gaussian weighted position of each point within a normalized window around a feature point $(4 x$-bins and $4 y$-bins) and the magnitude and phase response (4 phase orientation bins). 
TABle 1: ICE 2005 database matching results. *In the NIST ICE Phase 2005, Duagman 1 and Duagman 2 were listed as Cam 1 and Cam 2. The EERs are not clear to view from the report. The GAR at FAR $=01 \%$ and GAR at FAR $=0.01 \%$ were taken from the plot of the report in [30].

(a) Right eyes.

\begin{tabular}{lcccc}
\hline Algorithm & \#Images & EER & GAR at FAR $=.1 \%$ & GAR at FAR $=.01 \%$ \\
\hline Daugman 1* & 1426 & - & 0.9940 & 0.9910 \\
Daugman 2* & 1426 & - & 0.9950 & 0.9920 \\
2D Gabor & 1426 & 0.0062 & 0.9900 & 0.9850 \\
1D Log-Gabor & 1426 & 0.0079 & 0.9870 & 0.9735 \\
Regional SIFT & 1426 & 0.0557 & 0.7320 & 0.5640 \\
Proposed & 1426 & 0.0185 & 0.9588 & 0.9386 \\
\hline
\end{tabular}

(b) Left eyes.

\begin{tabular}{lcccc}
\hline Algorithm & \#Images & EER & GAR at FAR $=.1 \%$ & GAR at FAR $=.01 \%$ \\
\hline Daugman $1^{*}$ & 1527 & - & 0.9880 & 0.9850 \\
Daugman 2* & 1527 & - & 0.9890 & 0.9880 \\
2D Gabor & 1527 & 0.0126 & 0.9750 & 0.9629 \\
1D Log-Gabor & 1527 & 0.0106 & 0.9739 & 0.9533 \\
Regional SIFT & 1527 & 0.0689 & 0.6346 & 0.3741 \\
Proposed & 1527 & 0.0257 & 0.9316 & 0.8916 \\
\hline
\end{tabular}

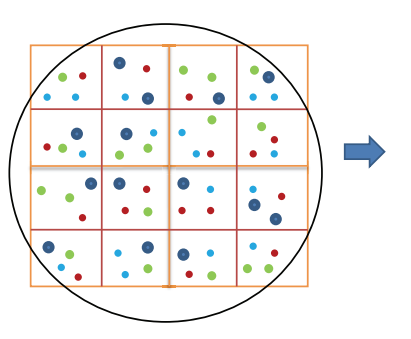

(a) Normalised window

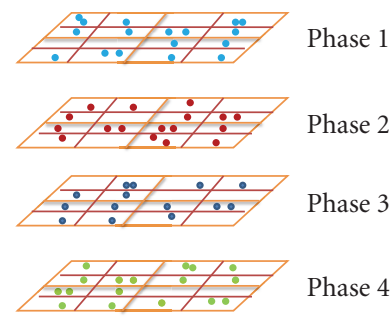

(b) Gabor descriptor
FIGURE 6: The process of a Gabor descriptor. The different colors in the left image shows that the Gabor filter results show they are in different phase layers.

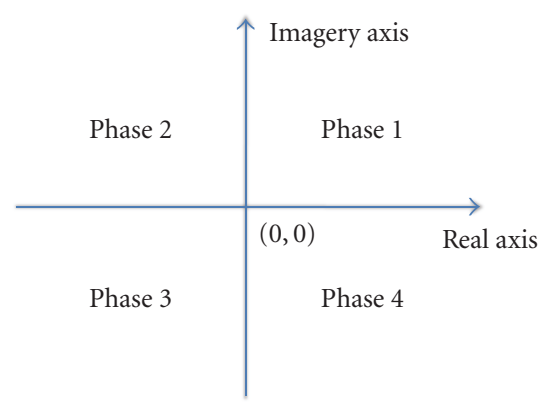

Figure 7: Phase areas.

For each feature point, we first choose its local window for feature description. The window size is determined as

$$
W=\left\lfloor\sqrt{2} \cdot S_{A} \cdot \frac{N+1}{2}+0.5\right\rfloor
$$

where $S_{A}=\left(\sqrt{\left(x-x_{p}\right)^{2}+\left(y-y_{p}\right)^{2}}\right) \cdot(2 \pi / 360) \cdot 5$ and $N$ is the number of bins used to describe the relative position of a point to a feature point (here $N=4$ ). $S_{A}$ is the spatial extension of the frame around the feature point $(x, y)$ in the angular direction, $\left(x_{p}, y_{p}\right)$ are the coordinates of pupil center. $S_{A}$ is used to normalize the window around that feature point and changes in size based on the distance between the feature point and pupil centre.

The coordinates of all pixels in the window are then normalized: the pixel $(x, y)$ is normalized as

$$
\begin{aligned}
\left(n_{x}, n_{y}\right)=( & \frac{\left(x-x_{s}\right) \cos \theta+\left(y-y_{s}\right) \sin \theta}{S_{R}} \\
& \left.\frac{-\left(x-x_{s}\right) \sin \theta+\left(y-y_{s}\right) \cos \theta}{S_{A}}\right) .
\end{aligned}
$$

$S_{R}$ is the spatial extension around the feature point in the radial direction, and is used to normalize the window around that feature point and changes in size based on the amount of dilation. $S_{A}$ is the same as what we defined in (11). $\left(x_{s}, y_{s}\right)$ is the feature point. $\theta$ is the angle between the line of the pixel and the feature point to the line of the feature point and the pupil center (Figure 4). It is used to orient the window around the feature point such that the same feature point in another image will be able to be matched despite differences in angular position with respect to the pupil center.

In order to capture the iris features around a given feature point, a bank of 2D Gabor filters are used:

$$
\begin{aligned}
G(x, y) & \\
= & \frac{1}{2 \pi \sigma \beta} \exp \left\{-\pi\left(\frac{\left(x-x_{0}\right)^{2}}{\sigma^{2}}+\frac{\left(y-y_{0}\right)^{2}}{\beta^{2}}\right)\right\} \\
& \times \exp \left[i\left(\xi_{0} x+v_{0} y\right)\right] .
\end{aligned}
$$




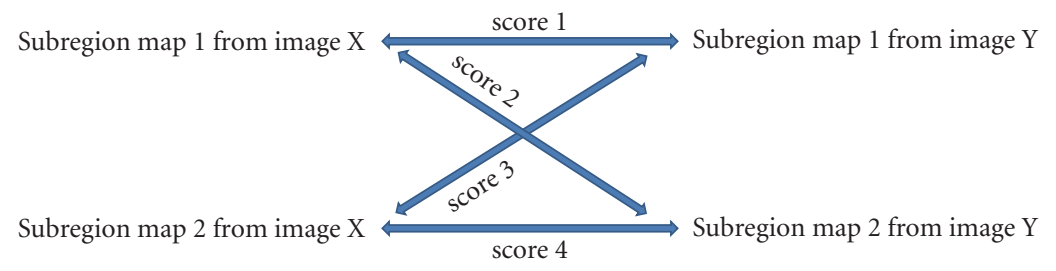

FIGURE 8: Subregion matching between two iris images.

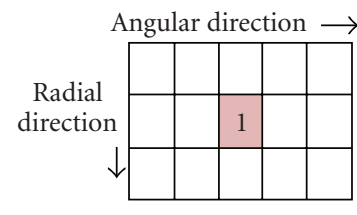

(a) Feature point 1 from image $X$
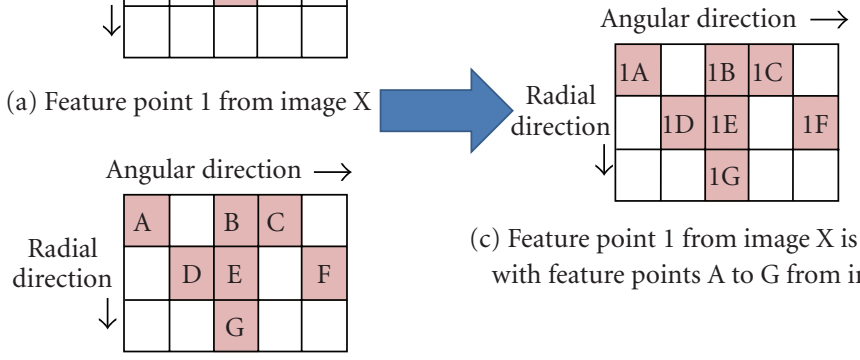

(b) The feature points that are located in the same location and neighbor locations from image $\mathrm{Y}$

Figure 9: Feature point matching.

In this equation, $\left(x_{0}, y_{0}\right)$ is the center of the receptive field of the spatial domain, $\left(\xi_{0}, v_{0}\right)$ is the frequency of the filter. $\sigma$ and $\beta$ are the standard deviations of the elliptical Gaussian along $x$ and $y$ directions. By properly designing these parameters, we can change the Gabor wavelet to fit the specific region for feature extraction. Figure 5 shows the example of Gabor odd and even filters in different sizes and orientations.

For each point in the normalized window around the feature point, the magnitude and phase response of the appropriate 2D Gabor wavelet is calculated with the wavelet centered on the point being considered. The magnitude is then Gaussian weighted based on the relative spatial distance from the feature point so that points in the window closest to the feature point carry the most weight and points further away carry less.

A Gabor descriptor is created by first computing the gradient magnitude and orientation phase at each point in a normalized window around the feature point location, as shown on Figure 6. These are weighted by a Gaussian window, indicated by the overlaid circle. These samples are then accumulated into four phase quadrics. In this paper, we separate the phase into 4 areas (Figure 7)

The weight of the Gaussian, wn, is calculated as

$$
w n=e^{-.5\left((n x)^{2} / 2 \sigma_{x}^{2}+(n y)^{2} / 2 \sigma_{y}^{2}\right)},
$$

where $\sigma_{y}=N / 2$ and $\sigma_{x}$ changes based on the dilation around the feature point. Finally, the weight of each point is calculated as

$$
\text { weight }=w n \cdot m g
$$

where $m g$ is the magnitude response of the 2D Gabor wavelet, and weight is added to one of 64 bins based on relative distance from the feature point and quantized phase response of the $2 \mathrm{D}$ Gabor wavelet. The resulting 64 bin feature point descriptor is then normalized to a unit vector by dividing by the 2-norm of the descriptor:

$$
\operatorname{descr}_{\text {norm }}=\frac{\text { descr }}{\|\operatorname{descr}\|_{2}} \text {. }
$$

Since each descriptor is normalized, the relative difference in magnitude response from the 2D Gabor filter remains the same for the same points around a feature point across iris images with different global illumination. And since phase is not affected by illumination, the same points in two iris images affect the same descriptor bins. Therefore, each feature point descriptor created has each of the 64 bins uniquely affected by the surrounding points based on distance from the feature point, and 2D Gabor wavelet response magnitude and phase; and an accurate descriptor is formed based entirely on the annular iris data.

3.4. Region-Based Matching. To match two iris images, the set of two 10 by 72 feature point maps are compared and the Euclidean distance is found between each feature point descriptor (Figure 8). In other words, the two feature point maps from image A are compared to the two feature point maps from image $\mathrm{B}$, resulting in 4 matching scores. The smallest matching score is then used as the matching score between the two images. Recall that the two feature point 


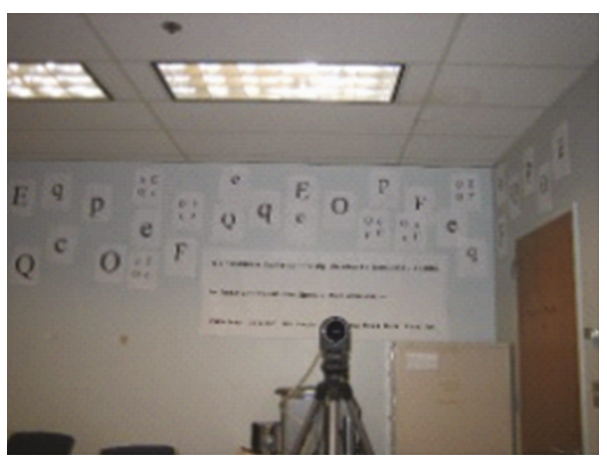

(a)

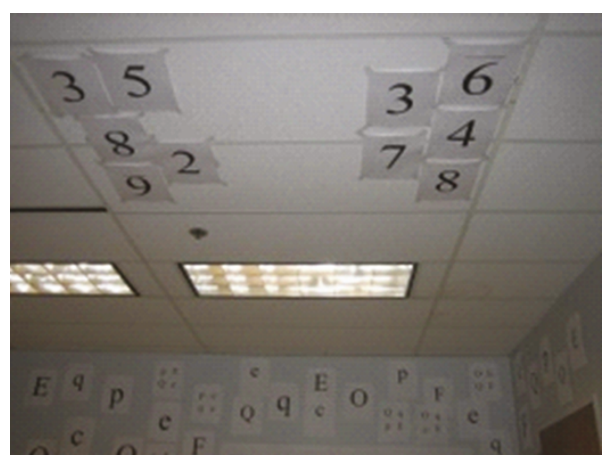

(b)

FIgURE 10: Remote Iris Image Acquisition Station Set Up.

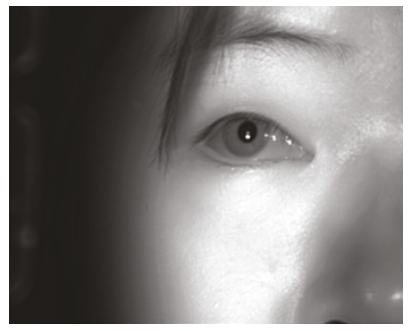

(a) Look Left

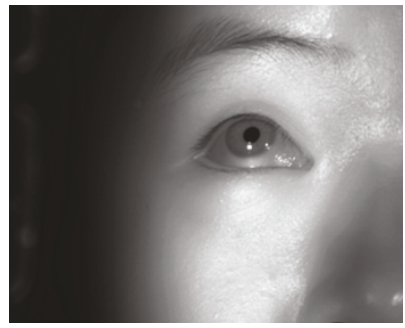

(d) Look Up-Left

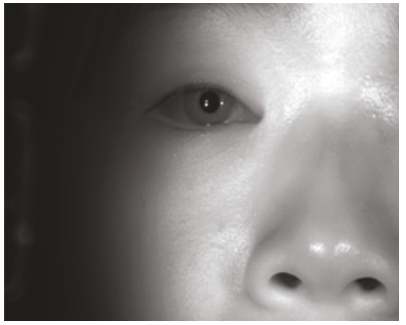

(b) Look Center

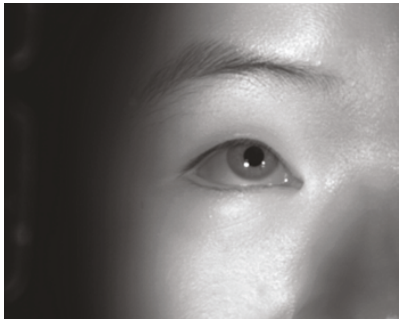

(e) Look Up

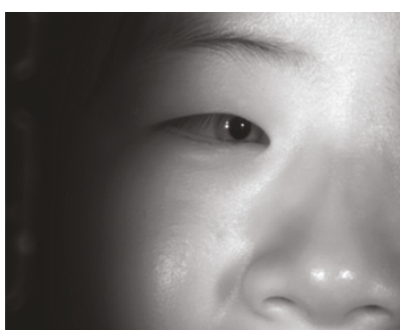

(c) Look Right

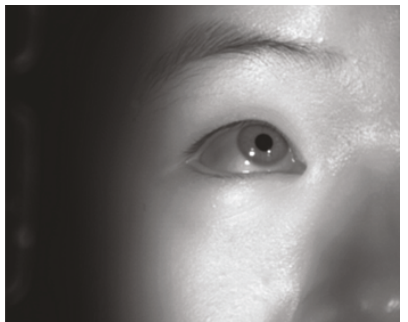

(f) Look Up-Right

Figure 11: IUPUI Remote Iris Image Database: Multiple Angles.

maps for an iris image describe the same regions, but are offset by half of the angular resolution of the bins. This is done in order to accommodate feature points that fall on boundaries of subregions within a feature point map.

To match two feature point maps, the average of the distance scores between all overlapping feature points is calculated and used as the matching score between two feature point maps. To make the proposed method tolerant of segmentation error and eye rotation, each feature point in a feature point map 1 is compared to each feature point in the fifteen surrounding bins (two bins on either side and one bin above and below) in a feature point map 2 (Figure 9), and the minimum average distance score is stored for the two feature point maps compared. In this way, the proposed method is less sensitive to the segmentation error that is prone to occur in nonideal iris images since feature points can occur anywhere within a bin and allowances are made to maximize the opportunity for the same two feature points in two images to be compared. Algorithms that sample the iris region and encode globally require more stringent segmentation results so as to correctly match each encoded point.

\section{Experimental Results}

4.1. ICE 2005 Database Results and Comparison. The ICE 2005 Database [30] from National Institute of Standards and Technology (NSIT) consists mostly of frontal look eyes. It includes 2 subdatabases: left iris image database with 1527 images from 120 subjects, and right iris image database with 1426 images from 124 subjects. In this experiment, we used the left eyes and right eyes, respectively, as ICE protocol in phase 2005 organized by NIST, and our goal was to compare the proposed method with traditional methods using only frontal eyes.

Table 1 shows the comparisons using different methods: Daugman's two methods called Cambridge 1 and Cambridge 2 methods [30], and our implementation of the traditional 2D Gabor wavelet matching and 1D Log-Gabor matching, 


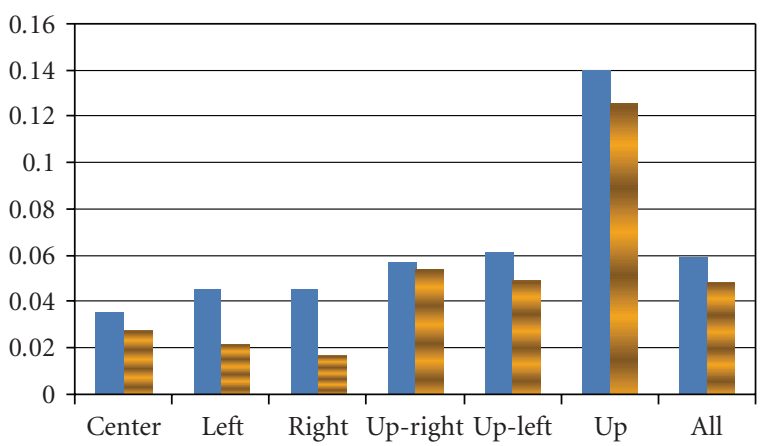

(a) Comparison using EER (the lower the better)

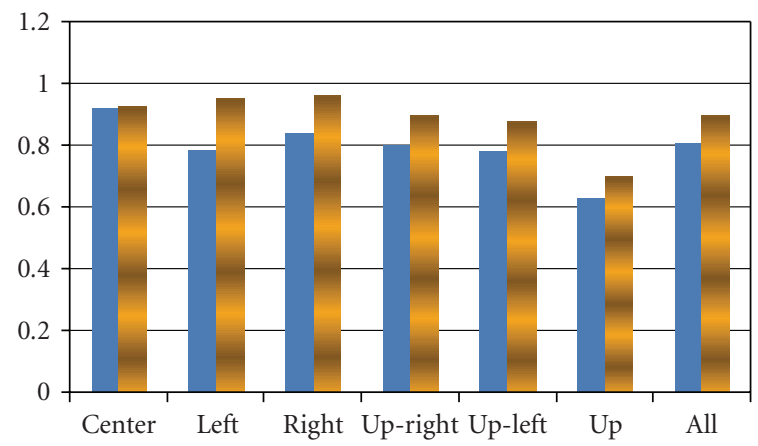

(b) Comparison using GAR at FAR $=0.1 \%$ (the higher the better)

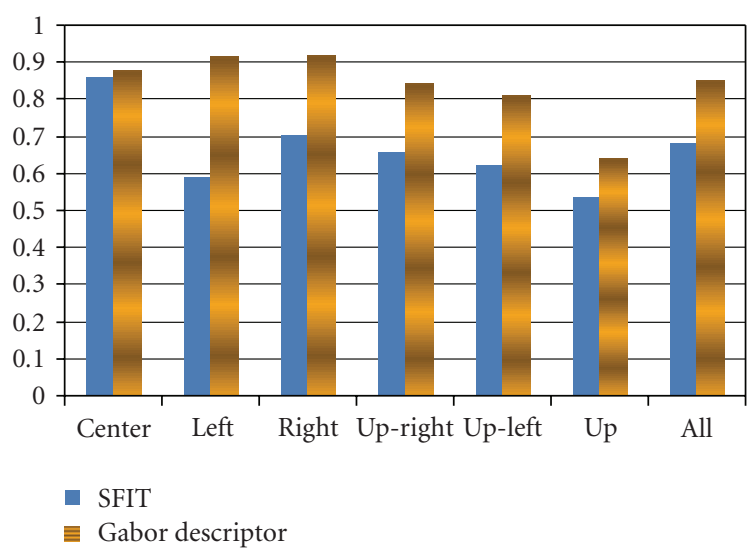

(c) Comparison using GAR at FAR $=0.01 \%$ (the higher the better)

FIGURE 12: Comparisons between the regional SIFT method and the proposed Gabor Descriptor method using EER, GAR at FAR = $0.1 \%$, and $\mathrm{GAR}$ at $\mathrm{FAR}=0.01 \%$.

our Regional SIFT, and the proposed method results on annular iris images. The two Cambridge results are the best in the list. It is unknown about the technical difference between the two Cambridge methods. They could use different segmentation methods or Gabor wavelet parameters. To be comparable, all our methods (our implementation of 2D Gabor wavelet, 1D Log-gabor wavelet, SIFT method, and the proposed method) used one segmentation method. It is shown that our implementation of the traditional methods obtains good results which are close to Daugman's results and our proposed method obtains comparable results. Note, Daugman's methods used his own segmentation approaches which are unknown to the public. The performance of Regional SIFT is understandable given the limitations previously mentioned. To reduce the effect of segmentation error to the traditional methods, manual segmentation was used to find the pupil and limbic boundaries, which were modeled as circles.

4.2. IUPUI Remote Iris Image Database Recognition Results and Comparison. The IUPUI Remote Iris Image Database was acquired at 10.3 feet from the camera to the subject using a MicroVista NIR camera with Fujinon zoom lens. 6 videos were captured for each subject with different scenarios: frontal look (1st video) reading from posters 15 feet from the subject and 5 feet behind the camera (2nd and 3rd videos) (Figure 10(a)); searching the wall to count the number of occurrences of a certain symbol (4th and 5th videos) (Figure 10(a)); performing simple calculations using numbers posted on the ceiling (6th video) (Figure 10(b)). Each video was acquired at 30 frames per second with $1280 \times$ 1024 resolution. The average iris radius of the video images in the database is 95 pixels. During the image acquisition, subjects can move their heads and eyes freely to perform the tasks, which simulates a remote, noncooperative situation, such as when a subject looks at flight times in an airport. In addition, the subjects can have their own emotions during the acquisition process (Some of the subjects smiled in some tasks). The authors are working with IRB to make this database available publically.

In this experiment, a database with 10 video frames for each iris for six classifications of angle with respect to the camera were constructed (Figure 11) from both sessions: looking center, left, right, up-left, up-right, and up. This resulted in 60 images per iris, with the exception that three iris videos were missing. The total number of images used for this experiment was 3690 and included both left and right eyes from 31 subjects (because 3 videos were incorrectly acquired and were not used in this paper).

\subsubsection{Frontal Look Recognition Results and Comparison.} Table 2 shows that our results using the proposed method and the Regional SIFT method are comparable to the results achieved using traditional matching on the centered eyes from our noncooperative database. The pupil and limbic boundaries were modeled as circles which is a simple and reasonable approximation of the pupil and limbic boundaries' geometries. We did not perform this same matching algorithm on the other classes since they are not frontal looking images and it would be difficult to reliably sample the iris pattern for off-angle images without some transformation such as Daugman proposed [2]. While this approach seems reasonable, we argue that due to the $3 \mathrm{D}$ nature of iris patterns, it is more reasonable to encode iris patterns without a transformation and more accurately represent the patterns presented to the camera.

4.2.2. Multiple Angle Recognition Results and Comparison. Table 3 shows the experimental results using the Regional SIFT method. Table 4 shows the experimental results using 


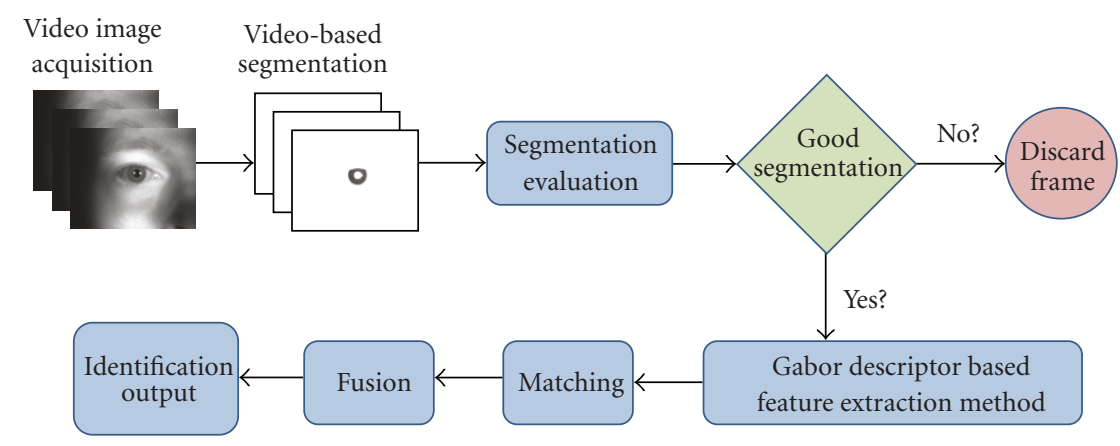

FIgURE 13: The Proposed video-based noncooperative iris recognition system.

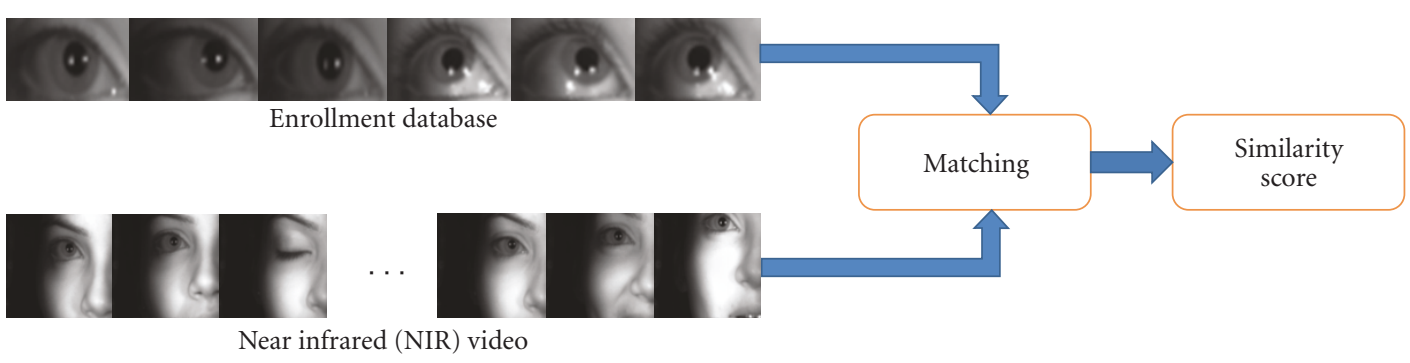

FIGURE 14: Matching protocol for noncooperative iris recognition.

TABLE 2: IUPUI remote database frontal look eyes matching results.

\begin{tabular}{lcccc}
\hline Algorithm & \#Images & EER & $\begin{array}{c}\text { GAR at FAR } \\
=.1 \%\end{array}$ & $\begin{array}{c}\text { GAR at FAR } \\
=.01 \%\end{array}$ \\
\hline 2D Gabor & 610 & 0.0179 & 0.9297 & 0.8856 \\
1D Log Gabor & 610 & 0.0295 & 0.9235 & 0.8980 \\
Regional SIFT & 610 & 0.0350 & 0.9173 & 0.8572 \\
Proposed & 610 & 0.0273 & 0.9213 & 0.8761 \\
\hline
\end{tabular}

the proposed method. Here all-to-all matching is used to match all irises. The genuine matches are the matching results from same eye with same-looking angles. The imposters are the matching results from different eyes with same- or different-looking angles. By comparing them (Figure 12), we see that the Regional SIFT method does not perform as well as the proposed method on the noncooperative iris images. The main reason is because Regional SIFT selects feature points using local gradient magnitude and angle information, whereas the proposed method encodes feature information around feature points using the magnitude and phase response of 2D Gabor wavelets which is more capable of capturing iris feature characteristics. In addition, the proposed method is less sensitive to segmentation error. The subregions of the proposed method are locally area adjusted to the iris dilation, contraction, and deformation.

For many methods, including the Regional SIFT method, center gaze would achieve better recognition accuracy than off-angle eyes. However, for the proposed method, our experiment results show that the left and right looking eyes have achieved higher accuracy than frontal looking images
TABLE 3: Recognition results of the regional SIFT method for same eyes divided into classes based on angle of gaze.

\begin{tabular}{lcccc}
\hline Classes & \#Images & EER & $\begin{array}{c}\text { GAR at FAR } \\
=.1 \%\end{array}$ & $\begin{array}{c}\text { GAR at FAR } \\
=.01 \%\end{array}$ \\
\hline Center & 610 & 0.0350 & 0.9173 & 0.8572 \\
Left & 620 & 0.0454 & 0.7800 & 0.5865 \\
Right & 620 & 0.0454 & 0.8340 & 0.6980 \\
Up-right & 600 & 0.0567 & 0.7941 & 0.6530 \\
Up-left & 620 & 0.0610 & 0.7725 & 0.6170 \\
Up & 620 & 0.1392 & 0.6265 & 0.5320 \\
All & 3690 & 0.0588 & 0.8024 & 0.6763 \\
\hline
\end{tabular}

(Figure 12 and Table 4). When the eye is looking left or right, the image resolution for one side of iris (left or right) would be reduced, but the resolution for another side of the iris is increased. This increased resolution of the iris pattern helps to select stable feature points in recognition. As a result, our method performs slightly better for left-looking or rightlooking iris images. This shows that the proposed method is well suited for use in a nonfrontal gaze situation.

\section{Video-Based Noncooperative Iris Recognition}

5.1. Proposed Video-Based Noncooperative Iris Recognition System. Figure 13 describes the proposed system which consists of acquiring video sequences of iris data, using videobased noncooperative iris image segmentation, evaluating segmentation results, retaining the best segmented images, 
TABLE 4: Recognition results of the proposed method for same eyes divided into classes based on angle of gaze.

\begin{tabular}{lcccc}
\hline Classes & \#Images & EER & $\begin{array}{c}\text { GAR at FAR } \\
=.1 \%\end{array}$ & $\begin{array}{c}\text { GAR at FAR } \\
=.01 \%\end{array}$ \\
\hline Center & 610 & 0.0273 & 0.9213 & 0.8761 \\
Left & 620 & 0.0214 & 0.9487 & 0.9108 \\
Right & 620 & 0.0162 & 0.9613 & 0.9180 \\
Up-right & 600 & 0.0540 & 0.8956 & 0.8422 \\
Up-left & 620 & 0.0492 & 0.8742 & 0.8079 \\
Up & 620 & 0.1251 & 0.6950 & 0.6358 \\
All & 3690 & 0.0478 & 0.8966 & 0.8476 \\
\hline
\end{tabular}

feature extraction using the proposed Gabor descriptor, feature matching, and fusion.

Matching Protocol. Under noncooperative situation, the iris images tend to have lower quality and can be off-angle. To ensure the accuracy, it will be important to have multiple enrollment images with different eye-looking angle. In this paper, we propose the matching protocol to be multiple enrollment images with input video image (Figure 14).

Video-Based Noncooperative Iris Image Segmentation. Since noncooperative iris images can be especially difficult to segment using traditional methods [3, 9, 27-34], the videobased noncooperative iris image segmentation algorithm developed in our lab [61] is used in this paper. It uses a course-to-fine approach and uses a general conic to model the pupil and limbic boundaries. More details of this method can be found in [61].

Segmentation Evaluation. The segmentation evaluation method developed in our lab in [32] was used in this paper to estimate the accuracy of the segmentation result.

Feature Extraction and Matching. The 10 images with the best segmentation scores are used for recognition. The proposed Gabor descriptor method (introduced in Section 3) was used for feature extraction and matching.

Score Fusion. The matching score between the enrollment image and the individual video frame is fused with the segmentation evaluation score. After majority vote, if the best matching score of the video to an enrollment iris satisfies the matching threshold, the matching score will be the matching result for the video to that enrollment eye. Matching results from the video sequence to other enrollment eyes will be set to 1 ( 1 means no match). If even the highest matching score does not satisfy the matching threshold, this video will not be matched to any eye.

5.2. Experimental Results and Discussion. In this experiment, 10 images per eye were used from the first session for enrollment. They include the different off-angles (left, right, up-left, up-right, and up). The total number of enrollment images is 620 with 62 irises from 31 subjects. We automatically match the enrollment images with the video frames in the 5 videos for each person from the second session (the frontal look only video was excluded as they are all frontal images) for 30 subjects and 60 irises. 1 subject did not have a second session and 2 subjects only had 4 videos from the second session. Totally, we have 298 video sequences.

The result is $\mathrm{FAR}=0$ and $\mathrm{EER}=0$ for all thresholds since only one or zero matching scores are retained for each video. 73 videos (about $24.5 \%$ of the videos) were not recognized since some videos could not generate satisfactory matching results. For the rest of the videos, there is $100 \%$ recognition accuracy ( $0 \%$ FAR at $0 \%$ FRR). The results show that $100 \%$ accuracy can be obtained using multiple enrollment images, video sequences of an iris, and fusion of matching scores; even in a noncooperative iris database.

\section{Conclusion}

In this paper, we proposed Gabor Descriptor-Based Noncooperative Iris Recognition. The proposed solution to noncooperative iris recognition does not transform the iris to polar coordinates, is normalized for changes in dilation/contraction/deformation, and is tolerant of the segmentation errors that are likely to occur in a noncooperative situation. Experimental results show that the proposed method is comparable to traditional methods on the ICE 2005 database [30] and performs well for the IUPUI Remote Iris Image database. Results also show that visible iris features change as the gaze of an iris changes and that video-based iris recognition can greatly improve recognition accuracy when multiple angle enrollment iris images are used.

\section{Acknowledgments}

The authors would like to gratefully thank Luke Thomas for his help in this project. The authors also would like to thank Professor J. R. Matey of the US Naval Academy for helpful discussions of his published papers on iris recognition in low constraint scenarios. They would also like to thank Mr. R. Kirchner from the Department of Defense for his help and support. They would also like to thank MicroVista for partial support of the camera equipment [62]. They would also like to thank the people who contributed their iris data for this project. The research in this paper uses the ICE database provided by NIST [30]. This project is sponsored by the ONR Young Investigator Program (Award no. N00014-071-0788).

\section{References}

[1] A. K. Jain, A. Ross, and S. Pankanti, "Biometrics: a tool for information security," IEEE Transactions on Information Forensics and Security, vol. 1, no. 2, pp. 125-143, 2006.

[2] Y. Du, "Review of iris recognition: cameras, systems, and their applications," Sensor Review, vol. 26, no. 1, pp. 66-69, 2006.

[3] K. W. Bowyer, K. Hollingsworth, and P. J. Flynn, "Image understanding for iris biometrics: a survey," Computer Vision and Image Understanding, vol. 110, no. 2, pp. 281-307, 2008. 
[4] A.K. Jain, P. Flynn, A. A. Ross, et al., Handbook of Biometrics, Springer, New York, NY, USA, 2008.

[5] H. Proenca and L. A. Alexandre, "Toward noncooperative iris recognition: a classification approach using multiple signatures," IEEE Transactions on Pattern Analysis and Machine Intelligence, vol. 29, no. 4, pp. 607-612, 2007.

[6] J. Daugman, "Statistical richness of visual phase information: update on recognizing persons by iris patterns," International Journal of Computer Vision, vol. 45, no. 1, pp. 25-38, 2001.

[7] J. Daugman, "How iris recognition works," IEEE Transactions on Circuits and Systems for Video Technology, vol. 14, no. 1, pp. 21-30, 2004.

[8] J. Daugman, "Probing the uniqueness and randomness of iriscodes: results from 200 billion iris pair comparisons," Proceedings of the IEEE, vol. 94, no. 11, pp. 1927-1934, 2006.

[9] J. Daugman, "New methods in iris recognition," IEEE Transactions on Systems, Man, and Cybernetics. Part B, vol. 37, no. 5, pp. 1167-1175, 2007.

[10] Y. Chen, S. C. Dass, and A. K. Jain, "Localized iris image quality using 2-D wavelets," in Proceedings of the IEEE International Conference on Biometrics, Hong Kong, China, 2006.

[11] J. R. Matey, O. Naroditsky, K. Hanna, et al., "Iris on the move: acquisition of images for iris recognition in less constrained environments," Proceedings of the IEEE, vol. 94, no. 11, pp. 1936-1946, 2006.

[12] L. Masek and P. Kovesi, MATLAB Source Code for a Biometric Identification System Based on Iris Patterns, University of Western Australia, Perth, Australia, 2003.

[13] L. Ma, T. Tan, Y. Wang, and D. Zhang, "Personal identification based on iris texture analysis," IEEE Transactions on Pattern Analysis and Machine Intelligence, vol. 25, no. 12, pp. 15191533, 2003.

[14] R. P. Wildes, "Iris recognition: an emerging biometrie technology," Proceedings of the IEEE, vol. 85, no. 9, pp. 1348-1363, 1997.

[15] N. Sudha, N. B. Puhan, H. Xia, and X. Jiang, "Iris recognition on edge maps," in Proceedings of the 2007 6th International Conference on Information, Communications and Signal Processing, Singapore, 2007.

[16] N. Sudha, N. B. Puhan, H. Xia, and X. Jiang, "Iris recognition on edge maps," IET Computer Vision, vol. 3, no. 1, pp. 1-7, 2009.

[17] W. W. Boles and B. Boashash, "A human identification technique using images of the iris and wavelet transform," IEEE Transactions on Signal Processing, vol. 46, no. 4, pp. 1185$1188,1998$.

[18] Z. Sun, Y. Wang, T. Tan, and J. Cui, "Improving iris recognition accuracy via cascaded classifiers," IEEE Transactions on Systems, Man and Cybernetics. Part C, vol. 35, no. 3, pp. 435441, 2005.

[19] K. P. Hollingsworth, K. W. Bowyer, and P. J. Flynn, “The best bits in an Iris code," IEEE Transactions on Pattern Analysis and Machine Intelligence, vol. 31, no. 6, pp. 964-973, 2009.

[20] J. Thornton, M. Savvides, and B. V. K. V. Kumar, "A Bayesian approach to deformed pattern matching of iris images," IEEE Transactions on Pattern Analysis and Machine Intelligence, vol. 29, no. 4, pp. 596-606, 2007.

[21] Y. Du, R. W. Ives, D. M. Etter, and T. B. Welch, "Use of onedimensional iris signatures to rank iris pattern similarities," Optical Engineering, vol. 45, no. 3, 037201, pp. 1-10, 2006.

[22] V. Velisavljevic, "Low-complexity iris coding and recognition based on directionlets," IEEE Transactions on Information Forensics and Security, vol. 4, no. 3, pp. 410-417, 2009.
[23] K. Miyazawa, K. Ito, T. Aoki, K. Kobayashi, and H. Nakajima, "An effective approach for Iris recognition using phase-based image matching," IEEE Transactions on Pattern Analysis and Machine Intelligence, vol. 30, no. 10, pp. 1741-1756, 2008.

[24] N. Tajbakhsh, B. N. Araabi, and H. Soltanianzadeh, "An intelligent decision combiner applied to noncooperative iris recognition," in Proceedings of the 11th International Conference on Information Fusion (FUSION '08), 2008.

[25] C. Belcher and Y. Du, "A selective feature information approach for iris image quality measure," IEEE Transactions on Information Forensics and Security, vol. 3, no. 3, pp. 572-577, 2008.

[26] Y. Du, C. Belcher, Z. Zhou, and R. W. Ives, "Feature correlation evaluation approach for iris image quality measure," Signal Processing, vol. 90, no. 4, pp. 1176-1187, 2010.

[27] H. Proenca and L. A. Alexandre, "Iris segmentation methodology for non-cooperative recognition," IEE Proceedings: Vision, Image and Signal Processing, vol. 153, no. 2, pp. 199-205, 2006.

[28] UBIRIS database, http://iris.di.ubi.pt/.

[29] CASIA database, http://www.cbsr.ia.ac.cn/IrisDatabase.htm.

[30] P. J. Phillips, K. W. Bowyer, P. J. Flynn, X. Liu, and W. T. Scruggs, "The iris challenge evaluation 2005," in Proceceedings of the IEEE 2nd International Conference on Biometrics: Theory, Applications and Systems, Arlington, Va, USA, 2008.

[31] M. Vatsa, R. Singh, and A. Noore, "Improving iris recognition performance using segmentation, quality enhancement, match score fusion, and indexing," IEEE Transactions on Systems, Man, and Cybernetics. Part B, vol. 38, no. 4, pp. 10211035, 2008.

[32] Z. Zhou, Y. Du, and C. Belcher, "Transforming traditional iris recognition systems to work in nonideal situations," IEEE Transactions on Industrial Electronics, vol. 56, no. 8, pp. 32033213, 2009.

[33] S. A.C. Schuckers, N. A. Schmid, A. Abhyankar, V. Dorairaj, C. K. Boyce, and L. A. Hornak, "On techniques for angle compensation in nonideal iris recognition," IEEE Transactions on Systems, Man, and Cybernetics. Part B, vol. 37, no. 5, pp. 1176-1190, 2007.

[34] C. Belcher and Y. Du, "Region-based SIFT approach to iris recognition," Optics and Lasers in Engineering, vol. 47, no. 1, pp. 139-147, 2009.

[35] K. Mikolajczyk and C. Schmid, "A performance evaluation of local descriptors," IEEE Transactions on Pattern Analysis and Machine Intelligence, vol. 27, no. 10, pp. 1615-1630, 2005.

[36] D. G. Lowe, "Distinctive image features from scale-invariant keypoints," International Journal of Computer Vision, vol. 60, no. 2, pp. 91-110, 2004.

[37] Y. Ke and R. Sukthankar, "PCA-SIFT: a more distinctive representation for local image descriptors," in Proceedings of the IEEE Computer Society Conference on Computer Vision and Pattern Recognition, vol. 2, pp. II506-II513, Washington, DC, USA, 2004.

[38] G. Carneiro and A. D. Jepson, "Flexible spatial configuration of local image features," IEEE Transactions on Pattern Analysis and Machine Intelligence, vol. 29, no. 12, pp. 2089-2104, 2007.

[39] H. Cheng, Z. Liu, N. Zheng, et al., "A deformable local image discriptor," Proceedings of the IEEE Computer Society Conference on Computer Vision and Pattern Recognition (CVPR '08), vol. 29, pp. 1-8, 2008.

[40] E. N. Mortensen, H. Deng, and L. Shapiro, "A SIFT descriptor with global context," in Proceedings of the IEEE Computer 
Society Conference on Computer Vision and Pattern Recognition (CVPR '05), vol. 1, pp. 184-190, San Diego, Calif, USA, 2005.

[41] L. Haibin and D.W. Jacobs, "Deformation invariant image matching," in Proceedings of the 10th IEEE International Conference on Computer Vision (ICCV'05), vol. 1, 2005.

[42] A. E. Johnson and M. Hebert, "Using spin images for efficient object recognition in cluttered 3D scenes," IEEE Transactions on Pattern Analysis and Machine Intelligence, vol. 21, no. 5, pp. 433-449, 1999.

[43] J. Assfalg, M. Bertini, A. Del Bimbo, and P. Pala, "Contentbased retrieval of 3-D objects using spin image signatures," IEEE Transactions on Multimedia, vol. 9, no. 3, pp. 589-599, 2007.

[44] S. Belongie, J. Malik, and J. Puzicha, "Shape matching and object recognition using shape contexts," IEEE Transactions on Pattern Analysis and Machine Intelligence, vol. 24, no. 4, pp. 509-522, 2002.

[45] G. Mori, S. Belongie, and J. Malik, "Efficient shape matching using shape contexts," IEEE Transactions on Pattern Analysis and Machine Intelligence, vol. 27, no. 11, pp. 1832-1837, 2005.

[46] G. Mori and J. Malik, "Recovering 3D human body configurations using shape contexts," IEEE Transactions on Pattern Analysis and Machine Intelligence, vol. 28, no. 7, pp. 10521062, 2006.

[47] W. T. Freeman and E.H. Adelson, "The design and use of steerable filters," IEEE Transactions on Pattern Analysis and Machine Intelligence, vol. 13, no. 9, pp. 891-906, 1991.

[48] E. P. Simoncelli and H. Farid, "Steerable wedge filters for local orientation analysis," IEEE Transactions on Image Processing, vol. 5, no. 9, pp. 1377-1382, 1996.

[49] M. Jacob and M. Unser, "Design of steerable filters for feature detection using Canny-like criteria," IEEE Transactions on Pattern Analysis and Machine Intelligence, vol. 26, no. 8, pp. 1007-1019, 2004.

[50] X. Shi, A. L. Ribeiro Castro, R. Manduchi, and R. Montgomery, "Rotational invariant operators based on steerable filter banks," IEEE Signal Processing Letters, vol. 13, no. 11, pp. 684-687, 2006.

[51] V. Lepetit and P. Fua, "Keypoint recognition using randomized trees," IEEE Transactions on Pattern Analysis and Machine Intelligence, vol. 28, no. 9, pp. 1465-1479, 2006.

[52] P. Quelhas, F. Monay, J.-M. Odobez, D. Gatica-Perez, and T. Tuytelaars, "A thousand words in a scene," IEEE Transactions on Pattern Analysis and Machine Intelligence, vol. 29, no. 9, pp. 1575-1589, 2007.

[53] T. Deselaers, L. Pimenidis, and H. Ney, "Bag-of-visualwords models for adult image classification and filtering," in Poceedings of the 19th International Conference on Pattern Recognition (ICPR '08), Tampa, Fla, USA, 2008.

[54] N. Lazic and P. Aarabi, "Importance of feature locations in bag-of-words image classification," in Poceedings of the IEEE International Conference on Acoustics, Speech and Signal Processing, vol. 1, pp. I641-I644, Honolulu, Hawaii, USA, 2007.

[55] T. Botterill, S. Mills, and R. Green, "Speeded-up bag-of-words algorithm for robot localisation through scene recognition," in Poceedings of the 23rd International Conference Image and Vision Computing New Zealand (IVCNZ '08), 2008.

[56] J.-H. Hsiao, C.-S. Chen, and M.-S. Chen, "A novel languagemodel-based approach for image object mining and reranking," in Poceedings of the IEEE International Conference on Data Mining, (ICDM '08), pp. 243-252, 2008.
[57] Z. Zhang, S. Chan, and L.-T. Chia, "Codebook+: a new module for creating discriminative codebooks," in Proceedings of the 2007 IEEE International Conference on Multimedia and Expo (ICME'07), pp. 815-818, 2007.

[58] L. Wu, S. Luo, and W. Sun, "Create efficient visual codebook based on weighted mRMR for object categorization," in Proceedings of the International Conference on Signal Processing (ICSP'08), pp. 1392-1395, 2008.

[59] F. Perronnin, "Universal and adapted vocabularies for generic visual categorization," IEEE Transactions on Pattern Analysis and Machine Intelligence, vol. 30, no. 7, pp. 1243-1256, 2008.

[60] Z. He, T. Tan, Z. Sun, and X. Qiu, "Toward accurate and fast iris segmentation for iris biometrics," IEEE Transactions on Pattern Analysis and Machine Intelligence, vol. 31, no. 9, pp. 1670-1684, 2009.

[61] Y. Du, E. Arslanturk, Z. Zhou, and C. Belcher, "Video-based non-cooperative iris image segmentation," IEEE Transactions on Systems, Man, and Cybernetics. Part B. In press.

[62] M. Cameras, http://www.intevac.com/intevacphotonics/products/microvista-nir. 